\title{
Descripción del ciclo anual de desarrollo del zooplancton de un lago de alta montaña mediante un análisis factorial
}

\author{
L. Cruz-Pizarro'; R. Morales ${ }^{2} ;$ A. González ${ }^{3}$
}

Departamento de Ecología. Fac. de Ciencias. Universidad de Granada. 2 Departamento de Zoología. Fac. de Ciencias. Universidad de Granada. 3 Departamento de Estadística. Fac. de Ciencias. Universidad de Granada.

\author{
SUMMARY \\ DESCRIPTION OF THE ANNUAL ZOOPLANKTON DEVELOPMENT CYCLE IN A
} HIGH MOUNTAIN LAKE BY A FACTORIAL ANALYSIS

The development of the zooplankton in the high mountain lake La Caldera (Andalucía, Spain) is described by a principal components analysis carried on data concerning population density for each zooplanktonic species as well as physical, chemical and biotic parameters measured during the ice-free period in 1977 from the July to the beginning of October).

A previous analysis using 21 variables and 16 sampling dates ( 16 cases, 21 variables) showed convenience in elimination of five of the se variables which caused singularity in the variance-covariance matrix. A second analysis, including 16 cases and 16 variables, allowed to establish that three factors account for more than $75 \%$ of the variance.

The three principal components are associated, respectively, with: 1st, temperature and development of the dominant species, both in number of individuals and time existence; 2nd, oxygen concentration, diversity and the "pioneer" species with short population development period; $3 \mathrm{rd}$, surface-bottom thermic differences and species whose coexistence mechanisms seems to be bases on a spatial segregation. An association of the sampling dates is proposed according to these three factors.

\section{INTRODUCCION}

La Caldera es un lago alpino de origen glaciar que se encuentra situado en la cara Sur de Sierra Nevada a $3.050 \mathrm{~m}$. de altitud. Sus características morfométricas, morfológicas $\mathrm{y}$, en parte, físico-químicas y bióticas ya han sido descritas (MARTINEZ, 1975, 1977), y en particular el estudio de la comunidad zooplanctónica durante los periodos libres de hielo de los años 1975 a 1977 ha sido objeto de un trabajo (CRUZ-PIZARRO, 1981) que ha dado a conocer su composición específica así como su evolucióri en el tiempo y en el espacio (distribución vertical).

El presente trabajo es una caracterización del ciclo anual de desarrollo del lago en función de las tendencias de variación más acusadas, puestas de manifiesto mediante análisis factorial a partir de las abundancias de las 
poblaciones zooplanctónicas presentes y de los valores de algunos parámetros físicoquímicos y bióticos cuya importancia ya ha sido puesta de manifiesto en el desarrollo de estas poblaciones. (CRUZ-PIZARRO, 1981).

\section{MATERIAL Y METODOS}

Los datos de partida utilizados son los obtenidos en los 16 muestreos realizados durante el año 1977. Las densidades de las poblaciones de cada especie, en cada una de las diferentes fechas, se obtuvieron al integrar los valores correspondientes a cada una de las distintas profundidades muestreadas, expresándose el resultado en org $/ \mathrm{m}^{2}$. Posterior. mente este valor sufrió una transformación $\log$ a.tmica al objeto de normalizar su distribución: $X_{i}^{\prime}=\log \left(X_{i}+1\right)$. Los valores de los parámetros físicos, químicos y biológicos en cada muestreo, corresponden al valor medio de los obtenidos a cada profundidad. También en este caso los datos sufrieron la misma transformación logarítmica, salvo aquéllos en los que intervienen logaritmos en su evaluación.

Para el cálculo de la diversidad hemos usado el índice de Shannon $\left(\mathrm{H}^{\prime}\right)$ :

$$
H^{\prime}=-\sum_{i=1}^{S} p_{i} \cdot \log _{2} p_{i}
$$

donde $p_{\mathbf{i}}$ es la proporción real de individuos $\left(\mathrm{N}_{\mathrm{i}} / \mathrm{N}\right)$ de la especie i y $\mathrm{S}$ es el número total de especies observadas.

La heterogeneidad vertical de la comunidad se ha obtenido como la diferencia entre el indice de diversidad calculado, en cada muestreo, para la columna de agua y el valor medio de la diversidad de la misma $\left(\overrightarrow{\mathrm{H}}^{\prime}\right)$ obtenido, siguiendo a MIRACLE (1976), según una expresión semejante a la utilizada en la integración del número de individuos, o sea:

$$
\bar{H}^{\prime}=\frac{1}{Z_{t}} \sum_{k=1}^{n}\left(\frac{Z_{k+1}-Z_{k-1}}{2}\right) \quad H_{k}^{\prime}
$$

donde $\mathrm{Z}_{\mathrm{k}}$ son las $\mathrm{n}$ profundidades muestreadas; $\mathrm{H}_{\mathrm{k}}{ }_{\mathrm{k}}$ es la diversidad a la profundidad $\mathrm{k} y$
$Z_{t}$ es la altura (en mts.) de la columna de agua muestreada en cad a caso.

Dentro del amplio abanico de posibilidades de creación de factores para reducir la dimensionalidad del problema se ha elegido el método del análisis factorial de componentes principales, debido a que la naturaleza de las variables observadas, transformadas en normales, permite elegir dentro de un vasto marco de interpretaciones estadísticas el significado real de los diferentes coeficientes (HARMAN, 1980), con la consiguiente ayuda a su interpretación biológica.

Por su amplia capacidad de cálculo y ya que es citado por diversos autores como el mejor (DRAPER \& SMITH, 1981) en el campo del análisis factorial, hemos utilizado el paquete de programas del BMDP en su versión 75, y más concretamente el programa P4M. Para eliminar el efecto debido a las diferentes magnitudes de cada variable se normalizaron trabajándose con la matriz de correlación, obtenida de la de varianzas-covarianzas.

\section{RESULTADOS}

Las tablas I y II recogen el número de individuos de cada especie obtenidos en cada muestreo y los valores de los parámetros físico-quimicos mencionados durante el año 1977.

Se realizó un primer análisis utilizando las 21 variables que aparecen en las tablas citadas. Consecuencia de dicho análisis fue la singularidad de la matriz de varianzas-covarianzas ( $y$ consecuentemente de la de correlación), esto es el determinante es nulo $|\Sigma|=0$, no existiendo por tanto inversa, en el sentido clásico, de esta matriz; lo cual obliga al uso de una matriz inversa generalizada $\Psi$ definida de la forma:

$$
\Sigma=\Psi \Sigma \Psi
$$

Ello es porque algún coeficiente de correlación múltiple de una variable con las demás es superior a 0.99999 (según el programa utilizado). Esto implica en la práctica que alguna variable depende linealmente de las 
TABLA I. Número de organismos (ind $/ \mathrm{m}^{2}$ ) observados en La Caldera durante el año 1977. 1. Mixodiaptomus laciniatus; 2: Diaptomus cyaneus; 3: Acanthocyclops vernalis; 4: Keratella cochlearis cochlearis; 5: $K$ c.tecta; 6: Hexarthra bulgarica; 7: Polyarthra remata; 8: P. major/vulgaris; 9: Filinia longiseta/terminalis; 10: Euchlanis dilatata; 11: Daphinia pulex; 12: Chydorus sphaericus; 13: Bosmina longirostris; 14: Codonella; 15: Difflugia.

\begin{tabular}{|c|c|c|c|c|c|c|c|c|c|c|c|c|c|c|c|}
\hline & 1 & $\dot{2}$ & 3 & 4 & 5 & 5 & 7 & 8 & $?$ & 10 & 11 & 12 & 13 & 14 & 15 \\
\hline $2 \varepsilon,-7$ & 21.88 & 187 & 250 & 1500 & 125 & -- & 375 & 250 & 750 & -- & $-\cdots$ & -- & 375 & 2313 & $\ldots$ \\
\hline $8-8$ & $968 ?$ & --- & 213 & 4000 & $-m$ & 438 & -- & -- & 313 & 138 & $-\infty$ & 63 & 375 & 5313 & 1938 \\
\hline $11-\varepsilon$ & 10056 & 250 & 2438 & 03 & -- & 250 & - & -- & -- & -- & -- & 534 & 219 & 3052 & 812 \\
\hline $6-8$ & 40253 & $-m$ & 250 & 8437 & 313 & 525 & 250 & -- & $-\ldots$ & - & $-\cdots$ & -- & 250 & 2313 & 2000 \\
\hline $19-8$ & 46570 & $-m-$ & 4751 & 213 & -- & 10313 & -- & --- & -- & 500 & -- & $1 \&:$ & -- & $2 E 13$ & $-\cdots$ \\
\hline $23-\xi$ & 112123 & 250 & 5562 & 31.3 & $-m$ & 21251 & $-\cdots$ & -- & 250 & 250 & -- & 213 & $--m$ & 41375 & 250 \\
\hline $20-\varepsilon$ & 51599 & $-m$ & 3000 & 9375 & 125 & 2812 & 4525 & 750 & 375 & - & 750 & 125 & 500 & 3313 & $-\cdots$ \\
\hline $30-\varepsilon^{2}$ & 119750 & -- & 5462 & 1313 & 63 & 10587 & 1503 & --- & 125 & --- & 63 & 63 & -- & 1187 & $-\cdots$ \\
\hline $2-9$ & 131451 & $\ldots$ & $382 \mathrm{E}$ & 8859 & -- & 6344 & 7765 & 1938 & 1297 & -- & 256 & 422 & $13 ?$ & 6312 & 750 \\
\hline $5-9$ & $154^{\prime} 738$ & -- & 1375 & 2313 & 5688 & 10875 & 1000 & 250 & 313 & -- & 250 & 125 & $-\infty$ & 2126 & 180 \\
\hline $9-0$ & 137372 & $5 \overline{3}$ & 375 & 1000 & -- & 1937 & 1313 & -- & 250 & $-m$ & 563 & 63 & --- & $\cdots$ & --- \\
\hline $12-9$ & 132801 & 375 & -- & -- & 5687 & 250 & $1 \varepsilon 8$ & -- & -- & 2000 & 250 & 1250 & -- & $-m$ & - \\
\hline $15-?$ & 130552 & -- & 1252 & -- & --- & 12329 & $46 ?$ & -- & 23,4 & 109 & 322 & 851 & -- & $93 E$, & -- \\
\hline $22-0$ & 135456 & $-m-$ & 1719 & -- & 125. & 20063 & - & -- & -- & $\Sigma \varepsilon \varepsilon$ & 94 & $46 \%$ & 158 & $-\cdots$ & $-\cdots$ \\
\hline $2 ?-?$ & $11480 \%$ & -- & --- & --- & -- & 4438 & $-m$ & -- & -- & 250 & man- & $43:$ & -- & -- & 53 \\
\hline $3-10$ & $10^{\prime} 1547$ & -- & 250 & -- & -- & 1.25 & -- & -- & $m$ & 94 & --- & 14.1 & $-m$ & 344 & $1 \geq 03$ \\
\hline
\end{tabular}

TABLA II. Valores de los parámetros físicos, químicos y bióticos considerados en La Caldera durante 1977. ts - tf: diferencias de temperatura superficie-fondo; Div.: diversidad; Het: heterogeneidad vertical.

$\begin{array}{rrrrrrr}\text { Fecha } & \begin{array}{c}\text { Oxígeno } \\ \text { mg/1 }\end{array} & \begin{array}{c}\text { Temperatura } \\ \text { oC }\end{array} & \begin{array}{c}\text { ts-tf } \\ \text { oC }\end{array} & \begin{array}{c}\text { Div. } \\ \text { bits/ind. }\end{array} & \text { Het. } & \begin{array}{r}\text { Prof. } \\ \max (\mathrm{m})\end{array} \\ 28-7 & 9,13 & 2,7 & 1,5 & 2,79 & 1,00 & 8,5 \\ 8-8 & 9,28 & 4,0 & 1,0 & 2,29 & 0,55 & 9,5 \\ 11-8 & 10,00 & 5,1 & 0,5 & 1,48 & 0,43 & 10,0 \\ 16-8 & 9,75 & 6,4 & 1,0 & 1,33 & 0,34 & 11,5 \\ 19-8 & 9,84 & 7,2 & 1,0 & 1,39 & 0,32 & 11,5 \\ 23-8 & 10,05 & 7,0 & 0,0 & 1,17 & 0,12 & 11,5 \\ 26-8 & 10,02 & 8,6 & 1,5 & 1,74 & 0,23 & 11,5 \\ 30-8 & 9,75 & 8,0 & 0,0 & 0,88 & 0,06 & 11,5 \\ 2-9 & 9,37 & 9,0 & 1,0 & 1,40 & 0,24 & 11,2 \\ 5-9 & 8,60 & 10,6 & 3,5 & 0,90 & 0,14 & 10,5 \\ 9-9 & 8,69 & 11,0 & 2,5 & 0,33 & 0,00 & 10,5 \\ 12-9 & 8,49 & 10,8 & 2,0 & 0,48 & 0,00 & 10,5 \\ 15-9 & 8,39 & 10,4 & 1,0 & 0,66 & 0,26 & 10,2 \\ 22-9 & 7,92 & 7,7 & 0,5 & 0,73 & 0,04 & 10,0 \\ 29-9 & 7,78 & 9,2 & 0,5 & 0,29 & 0,00 & 9,5 \\ 3-10 & 7,80 & 9,4 & 0,5 & 0,17 & 0,04 & 9,2\end{array}$


TABLA III. Porcentajes acumulados de la varianza explicada por los componentes con 21 y con 16 variables.

$\begin{array}{lcc} & 21 & 16 \\ & \text { variables } & \text { variables } \\ \text { Componente } & 29,6 & 34,4 \\ \text { Componente II } & 51,0 & 58,8 \\ \text { Componente III } & 67,0 & 74,9 \\ \text { Componente IV } & 73,5 & 81,9\end{array}$

restantes, por lo que se puede proceder a su eliminación en el resto del análisis.

Examinados dichos coeficientes de correlación múltiple asi como las correlaciones múltiples cuadráticas de cada una de las variables con los componentes principales (1o que nos da una medida de la explicación de cada variable conseguida con n componentes, siendo $\mathrm{n}<\mathrm{N}$ ), se decidió la eliminación de las siguientes: $D$, cyaneus, K.c. tecta, $P$. major/vulgaris, $B$. longirostris y la heterogeneidad vertical.

Efectuado el análisis sobre las 16 variables restantes, se pudo comprobar que, en cuanto al agrupamiento de fechas, el resultado de ambos análisis fue coincidente. La interpretación de los componentes, por el contrario, experimentó una mejora, como queda reflejado en la tabla III, alcanzando un nivel de casi el $75 \%$ de varianza explicada en el tercer componente, lo que permitirá una mejor interpretación biológica de estos tres componentes principales. La tabla IV muestra las cargas de las diferentes variables para cada uno de los tres primeros componentes.

\section{DISCUSION}

En el componente I las especies con mayor carga son: $M$. laciniatus, $H$. bulgarica y Ch. sphaericus. Variando conjuntamente con ellas aparece la temperatura con valores de carga muy elevados en este primer componente. Asimismo con valores elevados, pero negativos, aparece la diversidad. Son estas especies las que presentan una permanencia
TABLA IV. Cargas de las variables en cada uno de los tres primeros componentes.

\begin{tabular}{lrrr} 
& \multicolumn{3}{c}{ Componentes } \\
& I & II & III \\
M. laciniatus & 0,967 & $-0,211$ & 0,019 \\
A. vernalis & 0,097 & 0,847 & $-0,047$ \\
K.c. cochlearis & $-0,208$ & 0,749 & 0,497 \\
H. bulgarica & 0,860 & 0,260 & $-0,196$ \\
P. remata & 0,155 & 0,200 & 0,889 \\
E. longiseta & & & \\
terminalis & $-0,100$ & 0,499 & 0,497 \\
E. dilatata & 0,151 & $-0,403$ & $-0,736$ \\
D. pulex & 0,542 & $-0,041$ & 0,588 \\
Ch. sphaericus & 0,643 & $-0,142$ & $-0,431$ \\
Codonella sp.pl. & $-0,251$ & 0,813 & 0,033 \\
Difflugia sp.pl. & $-0,059$ & 0,127 & $-0,124$ \\
Oxigeno & $-0,126$ & 0,876 & 0,057 \\
Temp. media & 0,903 & $-0,321$ & 0,210 \\
Ts-Tf & $-0,086$ & $-0,266$ & 0,739 \\
Prof. máxima & 0,667 & 0,581 & 0,112
\end{tabular}

más continuada a lo largo de todo el periodo de estudio y además son las especies dominantes en cada uno de los grupos taxonómicos mayoritarios del zooplancton de $\mathrm{La}$ Caldera. Constituyen, pues, una fracción muy importante de la biomasa de la comunidad y el desarrollo de sus poblaciones, bien en cuanto a las variaciones en el número de individuos ( $M$. laciniatus) como en cuanto a su aparición ( $H$. bulgárica y en menor grado Ch. sphaericus), está estrechamente relacionado con la variación térmica del lago. Esto es especialmente evidente en el caso de $M$. laciniatus, la especie dominante en la comunidad, cuyo desarrollo se relaciona con la duración del período en que el lago se encuentra libre de hielo $y$, por ende, con la variación en la temperatura del agua (CRUZPIZARRO, 1981).

En general este componente podría repre- 


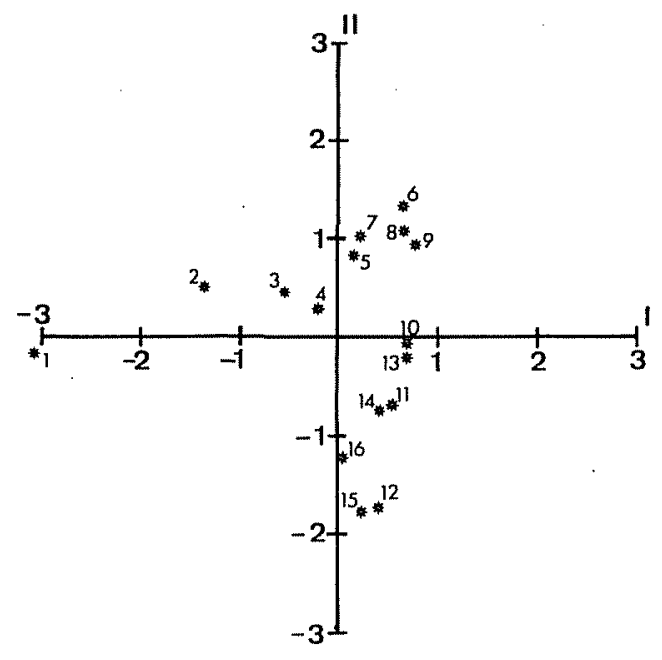

Fig. 1. Proyección de las fechas de muestreo en el plano definido por los dos primeros componentes principales.

sentar la variación de la temperatura que se correlaciona positivamente con el desarrollo de las especies dominantes, las cuales representan un porcentaje elevado de la biomasa de la comunidad y persisten durante la mayor parte del período de estudio por lo que la diversidad específica es casi siempre baja.

E1 segundo componente aparece gobernado por las especies $A$. pernalis, K.c. cochlearis y el Codonella así como por la concentración de oxígeno $\mathrm{y}$, en menor grado, por la diversidad. Se trata de organismos cuyo máximo de densidad poblacional aparece en los primeros muestreos, posteriores al deshie10 , en los que los valores de la diversidad son superiores a 1 bit/ind., por los altos valores de la uniformidad y de la riqueza especifica en estos momentos. Asociada con ellas se encuentra la concentración de oxígeno que igualmente es máxima en los momentos siguientes al deshielo. En resumen, este eje representa la explotación del medio por las especies pioneras cuya aparición coincide con las concentraciones de oxígeno más elevadas y determinan los máximos valores de diversidad.

El tercer componente viene definido por

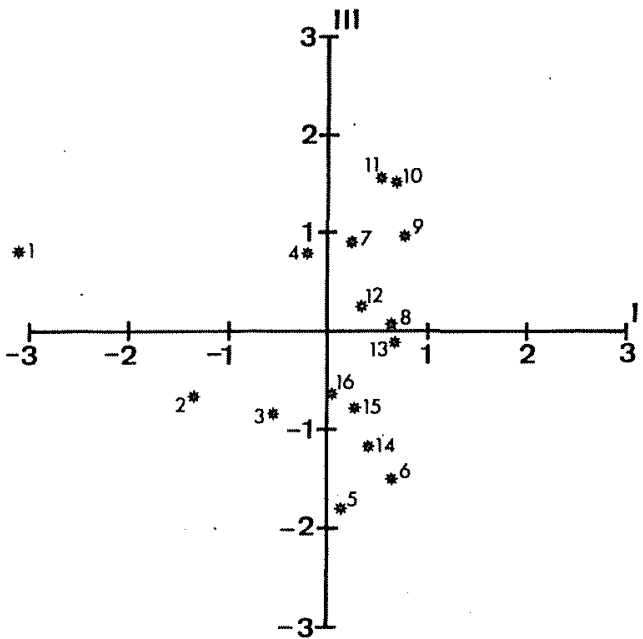

Fig. 2. Proyeccción de las fechas de muestreo en el plano definido por los componentes I y III.

las variables $P$. remata y $\mathrm{t}_{\mathrm{s}}-\mathrm{t}_{\mathrm{f}}$ (diferencia térmica superficie-fondo), aunque también tienen valores apreciables $D$. pulex, K.c. cochlearis y $F$. longiseta/terminalis. Se trata de especies, principalmente de Rotíferos, cuya presencia en la comunidad zooplanctónica se restringe (y, en todo caso, es máxima) a los momentos en que la diferencia térmica superficie-fondo es más acusada y cuyas poblaciones muestran una tendencia clara a situarse en los niveles superficiales más calientes $(D$. pulex muestra, en este sentido, un comportamiento algo diferente). Podríamos decir que este eje viene definido por las poblaciones cuyo mecanismo de coexistencia dentro de la comunidad parece estar basado en una segregación espacial. Es de notar que $E$. dilatata, que presenta una carga negativa muy acusada en este eje, muestra un período de existencia limitado prácticamente a los muestréos de mayor uniformidad térmica en profundidad.

La proyeccción de las fechas de muestreo en los ejes I y II (fig. 1), permite apreciar una separación entre los cuatro primeros muestreos (hasta el día 16-8-77) y el resto, en cuanto al eje I. Son los mómentos en los 
que las especies dominantes, y sobre todo M. laciniatus, aún no han alcanzado su máximo poblacional. En función del segundo componente, las fechas se pueden separar por el día noveno (2-9-77) que marca el final de la explotación del medio por las especies pioneras.
Finalmente, en función del tercer componente (fig. 2), hay que señalar la agrupación de puntos que se produce entre los muestreos 7 y 12 , que corresponde con el período de máxima segregación espacial de las poblaciones y de mayor diferencia térmica vertical. Un resultado semejante ha sido obtenido por MIRACLE (1974).

\section{BIBLIOGRAFIA}

BIOMEDICAL PROGRAM (BMDP). 1979. Institute of Health Sciences

CRUZ-PIZARRO, L. 1981. Estudio de la comunidad zooplanctónica de un lago de alta montaña (La Caldera, Sierra Nevada, Granada). Tesis Doctoral. Universidad de Granada. 180 págs.

DAGET, J. 1976. Les modèles mathématiques en écologie. Masson. París.

DRAPER, N.R. \& H. SMITH. 1981. Applied Regression Analysis. J. Wiley and son. Nueva York.

HARMAN, H.H. 1980. Análisis factorial modemo. Saltés. Madrid.

MARTINEZ, R.M. 1975. First report on the limno- logy of the alpine lake La Caldera, in the Penibetic Mountains (Sierra Nevada, Granada, Spain). Verh. Internat. Verein. Limnol, 19: 1.133-1.139.

MARTINEZ, R.M. 1977. Phytoplankton species, biomass and diversity in lake La Caldera (Sierra Nevada, Granada, Spain). Acta Hydrobiol. 19 (2): 95-107.

MIRACLE, M.R. 1974. Niche structure in freshwater zooplankton: a principal components approach. Ecology 55 (6): 1.306-1.316.

MIRACLE, M.R. 1976. Distribución en el espacio $y$ en el tiempo de las especies del zooplancton del lago de Banyoles. ICONA monografías no 5 . 